\title{
Potential Response of Passive Metal Electrodes to Free Chlorine in tap water
}

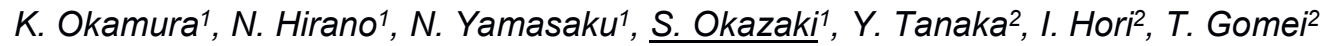 \\ 1 Graduate School of Engineering, Yokohama National University, Yokohama City, Japan, \\ ${ }^{2}$ R\&D, Aichi Tokei Denki. Co., Ltd., Nagoya City, Japan \\ okazaki-shinji-yp@ynu.ac.jp
}

\begin{abstract}
:
For realizing an all-solid-state free chlorine sensor composed of two dissimilar electrodes, dependences of open-circuit potentials on free chlorine concentration in simulated tap water condition were evaluated for several metal electrodes. In this study, stainless steel, tungsten, tantalum, and titanium which are well known metals to form stable passive film on its surface were tested. Although each metal electrode showed different sensitivity, it was found that the electrodes can detect free chlorine in practical concentration range below $1 \mathrm{mg} / \mathrm{L}$. Among them, stainless steel electrode exhibited relatively high sensitivity. This suggests that the appropriate combination of dissimilar metal electrodes enable us to measure free chlorine with high sensitivity and low cost.
\end{abstract}

Key words: all-solid-state potentiometric sensor, passive metal electrode, mixed potential, residual chlorine, tap water quality monitoring.

\section{Background}

In order to prevent various waterborne infections, water chlorination is an important process in drinking water treatment. Therefore, the free chlorine concentration is an important control parameter for safe water supply. The free chlorine concentration is mainly measured at water treatment plant and some related facilities. The number of monitoring point in whole water pipeline system is limited due to the lack of low cost and maintenance-free type sensor. By realizing such sensor device, detailed monitoring of free chlorine level at each water outlet over a wide area would be possible. Furthermore, integrating data collecting system using sophisticated telemetry techniques would enable us to control chlorine dosage efficiently and assure safety of drinking water network. Various analytical techniques for determining free chlorine concentration quantitatively have been proposed and commercialized. Among them, electrochemical methods have many advantages of non-reagent type, simplicity, low cost. They are easily applied to on-line monitoring. Although numerous amperometric sensors have been reported [1, 2], potentiometric-type sensors would be attractive candidate for realizing miniaturization, robustness, easy operation, low cost, and low power consumption. Recently, the authors have recently proposed an all-solid-state sensor composed of two dissimilar electrodes, each having a different sensitivity to free chlorine for the in-line monitoring of residual chlorine in tap water [3]. In this study, the characteristics of open circuit potential response of various passive metal electrodes were investigated and the sensitivities were compared in order to optimize this sensor configuration.

\section{Experimental Setup}

The open circuit potentials of several kinds of passive metal electrodes (stainless steel, tungsten, titanium, and tantalum) were measured against a saturated silver-silver chloride reference electrode by using a highinput impedance electrometer equipped in a conventional potentiostat/galvanostat. The electrode surfaces were polished with SiC paper (1200 grit) and ultrasonically cleaned with ethanol for $5 \mathrm{~min}$ and the electrodes were always stored in distilled water except for the measurements. Prior to the measurements, tested electrode was dipped in a tap water treated by an activated carbon filter to remove residual chlorine for about one day. A sodium hypochlorite solution was added to this test solution to have different free chlorine concentrations. The concentration of free chlorine was determined by portable photometer based on conventional colorimetry (DPD method). The solutions were continuously stirred by magnetic stirrer. All experiments were conducted at room temperature under atmospheric pressure. 


\section{Results and Discussion}

Fig. 1 shows the potential responses of SUS316 and pure titanium electrodes through successive addition of free chlorine. In the case of SUS316 electrodes, almost no response was observed below $0.05 \mathrm{mg} / \mathrm{L}$. However, the potential clearly responded to free chlorine in the concentration range above $0.19 \mathrm{mg} / \mathrm{L}$ although the response speed was relatively slow. On the other hand, titanium electrode could respond to extremely low concentration of free chlorine. After that, the response tended to saturate. In both cases, positive shifts caused by increasing of free chlorine concentration were observed. These response kinetics can be elucidated by mixed potential theory. Since passive metal electrodes were assessed in this study, the anodic reactions rate would be limited by passive currents which are necessary for maintaining protective oxide film formed on metal surface. On the other hand, the cathodic reactions consist of reduction reactions of free chlorine and dissolved oxygen. Under the open circuit condition, the passive current is balanced with the total cathodic current. The cathodic reaction is accelerated with increasing residual chlorine concentration. It results in shift of mixed potential in positive direction.

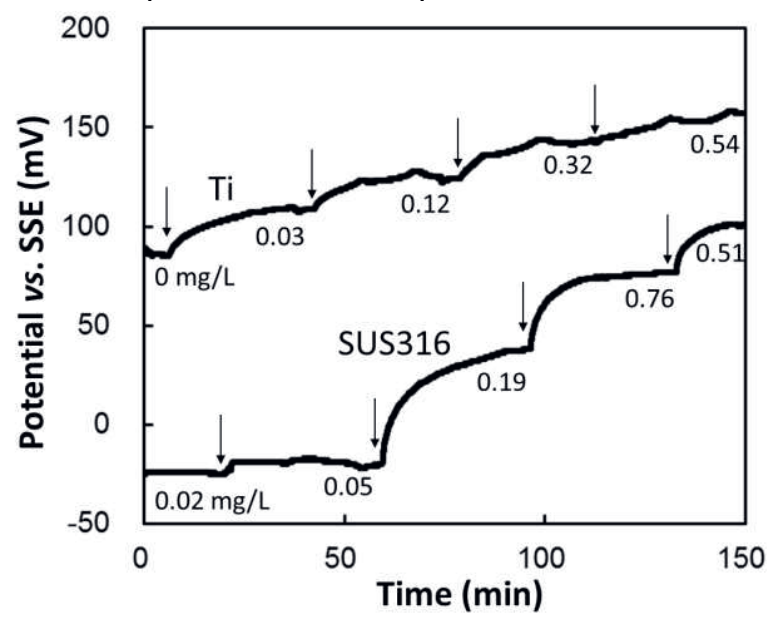

Fig. 1. Potential responses of stainless steel and titanium electrodes to free chlorine.

In order to clarify the concentration dependence of both electrode quantitatively, the open circuit potentials are plotted on Fig. 2. In the concentration range above $0.05 \mathrm{mg} / \mathrm{L}$, the potential is linearly related to logarithm of concentration. The value of slope of SUS316 and titanium are about 120 and $60 \mathrm{mV} /$ decade, respectively. This difference in sensitivity would be attributed to electrocatalytic properties against cathodic reaction of free chlorine or change in physicochemical condition of the passivation films. Although the data are not shown, a tantalum electrode was found to exhibit high sensitivity in low concentration range and tungsten electrode was relatively immune to free chlorine concentration.

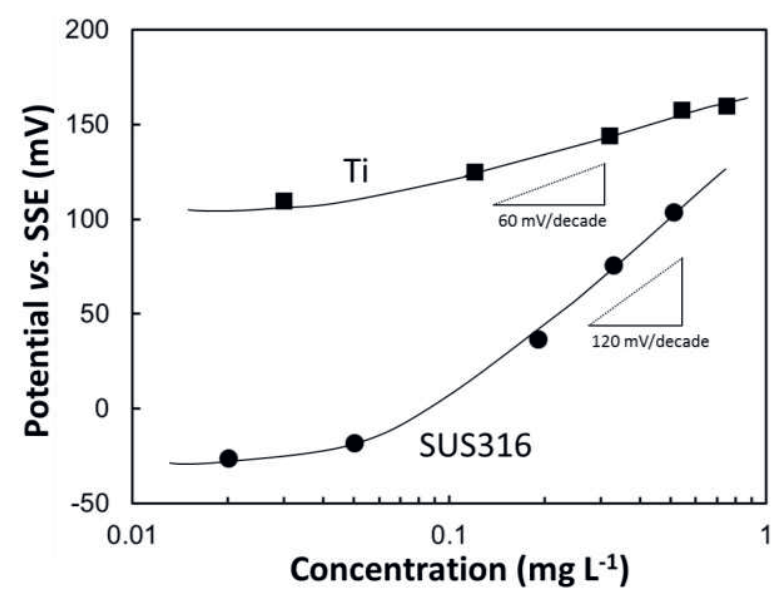

Fig. 2. Concentration dependence of open circuit potentials of stainless steel and titanium electrodes.

\section{Conclusion}

Two-electrode potentiometric sensor which is composed of solid-state electrodes could be easily miniaturized. Furthermore, it is also easy to realize wireless sensor system since the electric circuit for the operation is quite simple. This type of sensor is suitable for continuous inline monitoring of residual chlorine in pressurized tap water although the long term stability of open circuit potential should be improved.

\section{References}

[1] P. Salazar, M. Martín, F. J. García-García, J. L.González-Mora, A. R. González-Elipe, A novel and improved surfactant-modified Prussian Blue electrode for amperometric detection of free chlorine in water, Sensors and Actuators B: Chemical 213, 116-123 (2015); doi: 10.1016/j.snb.2015.02.092.

[2] S. Pan, M. J. Deen, R. Ghosh, Low-cost graphitebased free chlorine sensor, Anal. Chem. 87, 10734-10737 (2015); doi: 10.1021/acs.analchem.5b03164

[3] N. Kato, N. Hirano, S. Okazaki, S. Matsushita, T. Gomei, Development of an all-solid-state residual chlorine sensor for tap water quality monitoring, Sensors and Actuators B: Chemical 248, 10371044 (2017); doi: 10.1016/j.snb.2017.03.089 